\title{
Additional chemotherapy improved local control and overall survival after stereotactic body radiation therapy for patients with oligo-recurrence
}

Masaki Nakamura ${ }^{1,2^{*}}$ (D), Naoki Hashimoto ${ }^{1}$, Hiroshi Mayahara ${ }^{1}$, Haruka Uezono ${ }^{3}$, Aya Harada ${ }^{1}$, Ryo Nishikawa ${ }^{4}$, Yoshiro Matsuo ${ }^{5}$, Hiroki Kawaguchi ${ }^{1}$ and Hideki Nishimura ${ }^{1}$

\begin{abstract}
Background: Oligo-recurrence has been considered to confer improved prognosis than other oligometastatic conditions, and stereotactic body radiation therapy (SBRT) is considered as an option of local therapy for lung or liver metastases. The purpose of this study was to investigate the efficacy and safety of SBRT for lung and liver oligo-recurrent lesions and evaluate predictive factors for local control and prognosis.

Methods: This retrospective study included patients who presented with 1-3 matachronous lung or liver metastases, and treated with SBRT between May 2013 and March 2016 at a single institution. All patients harbored a controlled primary lesion. Patients with $<6$ months of follow-up were excluded. Local control, progression free survival, and overall survival rates were analyzed according to the Kaplan-Meier product limit method. Univariable log-rank and multivariable Cox regression analyses were performed to clarify predictive factors for local control and prognosis. Toxicity was graded according to the Common Terminology Criteria for Adverse Events, version 4.0.

Results: Seventy-six patients with a total of 70 and 44 lung and liver lesions were included. The median follow-up period was 21 (range, 7-43) months. The 1-year local control, progression-free survival and overall survival rates were 89,38 and $96 \%$, respectively. Smaller gross tumor volume and additional chemotherapy after SBRT were significant predictive factors for better local control $(p=0.005$ and $p=0.047)$, and the presence of a single metastatic lesion was a significant factor of good progression free survival $(p=0.008)$. Additional chemotherapy after SBRT was not a significant predictive factor but conferred to better overall survival $(p=0.078)$. Among colorectal cancer patients, post SBRT chemotherapy was significantly associated with better OS $(p=0.025)$. Over grade 3 adverse event was seen in only one patient.

Conclusion: SBRT is a safe and effective treatment for patients with lung and liver oligo-recurrence. Additional chemotherapy after SBRT improved local control, and single metastatic lesion was a significant predictive factor of better PFS in this study. Among colorectal cancer patients, additional chemotherapy after SBRT significantly associated better OS.
\end{abstract}

Keywords: Oligo, Meta, Recurrence, SBRT, Lung, Liver

\footnotetext{
* Correspondence: masanaka@east.ncc.go.jp

${ }^{1}$ Department of Radiation Oncology, Kobe Minimally invasive Cancer Center,

8-5-1, Minatojima Nakamachi, Chuo-ku, Kobe, Hyogo 650-0046, Japan

${ }^{2}$ Division of Radiation Oncology and Particle Therapy, National Cancer Center

Hospital East, 6-5-1, Kashiwanoha, Kashiwa, Chiba 277-8577, Japan

Full list of author information is available at the end of the article
}

(c) The Author(s). 2018 Open Access This article is distributed under the terms of the Creative Commons Attribution 4.0 International License (http://creativecommons.org/licenses/by/4.0/), which permits unrestricted use, distribution, and

reproduction in any medium, provided you give appropriate credit to the original author(s) and the source, provide a link to the Creative Commons license, and indicate if changes were made. The Creative Commons Public Domain Dedication waiver (http://creativecommons.org/publicdomain/zero/1.0/) applies to the data made available in this article, unless otherwise stated. 


\section{Background}

The condition of distant metastases from cancer has generally been regarded as a systemic disease [1], and the role of local therapy remains questionable. The condition of oligometastases, wherein the number and sites of metastatic tumors are limited [2], has been recently highlighted in the treatment of metastatic tumors. Metastatic disease is a leading cause of cancer mortality, but localized forms of cancer treatment may be effective in patients with oligometastases [3].

Oligo-recurrence is defined as the state in which patients with cancer have $\leq 5$ metastatic or recurrent lesions with controlled primary lesion, and has been considered to confer improved prognosis than syncoligometastases have $\leq 5$ metastatic or recurrent lesions with active primary lesions [4]. However, information on the specific oligometastatic cases in which local treatment may be effective remains insufficient and the selection of adaptation cases for local treatment is of particular concern to oncologists.

The lungs and liver are common sites for metastatic seeding, and surgery is a standard treatment option, with good results in terms of local control and survival $[5,6]$. Stereotactic body radiation therapy (SBRT) is a method for delivering high doses of radiation to the target, utilizing a small number of fractions with a high degree of precision within the body. It is an option for local therapy, and phase II studies utilizing this therapy for lung or liver metastases have reported good local control (LC) $[7,8]$.

In this study, we aimed to investigate the efficacy and safety of SBRT for lung and liver oligo-recurrent lesions and evaluate predictive factors for LC and prognosis.

\section{Methods}

This study included patients who were treated with SBRT between May 2013 and March 2016 at the Kobe Minimally Invasive Cancer Center. The study cohort included patients who presented with 1-3 metachronous lung or liver metastases with a controlled primary lesion. Patients with $<6$ months of follow-up were excluded. Pretreatment diagnosis was performed using computed tomography (CT), magnetic resonance (MR) imaging, or ${ }^{18}$ F-fluorodeoxyglucose positron emission tomography scans (FDG-PET). Histological proof of metastatic lesions was not indispensable. All study participants provided informed, written consent. The study protocol was approved by the Research Ethics Committee of our institution (reference number: 2018-[kenkyu01]-09). The research was conducted in accordance with the 1964 Declaration of Helsinki and its later amendments.

All SBRT procedures were performed with the CyberKnife $^{\bullet}$ VSI $^{\mathrm{Im}}$ System (Accuracy Inc., Sunnyvale, CA, USA). The CyberKnife SBRT method for lung tumors was performed as previously described [9]. A spine tracking system was used during the treatment of tumors that were located in the apical region and exhibited small respiratory movement. The spine tracking system is able to detect and track the bony anatomy of the spine to guide beam targeting without synchronizing respiratory movement. A directed tumor tracking system was used during the treatment of tumors that were over $15 \mathrm{~mm}$ in diameter, located in the visible in the orthogonal X-ray images created by the CyberKnife VSI System. Other tumors were treated with fiducial tracking system. In this system, the intravascular or transcutaneous method was used to place one fiducial marker close to the tumor. The motion of red light-emitting diodes attached to the patient chest wall was then registered and correlated to the location of the implanted fiducial, as determined by a series of orthogonal X-ray images taken during respiration.

The patients were immobilized with a Vac-Lok cushion (CIVCO, USA). A thin-sliced, four-dimensional CT scan was acquired with 1-mm slices. Contrast enhancement was performed as required. In the treatment of liver lesions, contrast-enhanced MR images were indispensable unless contraindicated. The organs at risk (i.e., the spinal cord, normal lung or liver tissue, heart, duodenum, stomach, kidney, and esophagus) were contoured on the CT scan at the resting respiratory level. Gross tumor volumes (GTVs) were contoured on each phase of the four-dimensional CT scans registered with the fiducial marker in the fiducial tracking system, the tumor in the tumor tracking system, and the vertebral body in the spine tracking system. The internal target volume was defined as a fusion of all GTVs at each phase of the four-dimensional CT scans. For lung tumors, the planning target volume (PTV) equaled the internal target volume plus 2-6 $\mathrm{mm}$; this heterogeneous margin was based on tracking methods. For liver tumors, PTV equaled the internal target volume plus $4 \mathrm{~mm}$; all liver tumors were treated with fiducial tracking methods. The MultiPlan 4.6.0 treatment planning software (Accuracy Inc., Sunnydale, CA, USA) was used to plan the treatments. Radiation doses were calculated using the Monte Carlo algorithm in lung tumors and the ray tracing algorithm in liver tumors. Treatment consisted of a 6-MV radiation beam using one or two circular collimator cones. The prescribed radiation dose delivered to the PTV was prescribed to the $75-85 \%$ isodose line, covering $\geq 95 \%$ of the PTV. The standard prescription dose was 60 Gy at our institute. However, in order to respect the constraints of the organs at risk, an adjustment of prescription dose was permitted. For patients with lung lesions, 3 or 4 fractionated therapy was performed. If PTV overlapped the hilar pulmonary vein or main bronchus, 8 fractionated therapy was selected. For patients 
with liver lesions, 4 fractionated therapy was performed. If PTV overlapped the right or left portal vein, 8 fractionated therapy was selected.

Patients initially underwent a follow-up CT 23 months following the SBRT. All patients underwent serial CT at 2-3-month intervals. Additional imaging, such as MR imaging or FDG-PET, was also performed if clinically indicated. All patients were registered until death or loss to follow-up. Local recurrence of lesions was defined as the evidence of tumor volume enlargement or the appearance of a new lesion in the PTV. Progression-free survival (PFS) was defined as the absence of local, regional, or distant progression. Suspected recurrence or new lesions were confirmed by at least two imaging modalities.

Local control, PFS, and overall survival (OS) rates were analyzed according to the Kaplan-Meier product limit method with 95\% confidence intervals (CIs) and were measured from the date of radiotherapy initiation. Univariable log-rank and multivariable Cox regression analyses were performed. Cut-off values of continuous valuables were calculated by receiver-operating characteristic curve (ROC) analyses. The Cox regression analysis simultaneously included factors that had shown associations $(p<0.100)$ in the log-rank analyses. When faced with factors that were correlated with each other, we selected the factor with the highest area under the curve in ROC analyses. All statistical analyses were conducted using $\mathrm{R}$ software, version 3.4.0 (The $\mathrm{R}$ Foundation, Vienna, Austria). All tests were two-sided and $p$-values of $<0.05$ were considered statistically significant. Toxicity was graded according to the Common Terminology Criteria for Adverse Events, version 4.0.

\section{Results}

Eighty-five patients who were treated with SBRT for oligo-recurrent lung and liver lesions between May 2013 and March 2016 at our institute were included. Nine patients were excluded because of loss to follow-up within 6 months. In total, 76 patients with a total of 70 lung lesions and 44 liver lesions were included. There was no patient whose metastatic lesions left untreated. Patient and lesion characteristics are provided in Table 1. Primary tumors in the patients were observed in the colorectum ( $n=46$ patients; $61 \%)$, gastric region $(n=5$ patients; 7\%), ovaries ( $n=2$ patients; $2.5 \%)$, esophagus $(n=2)$, pancreas $(n=2)$, lung $(n=2)$, liver $(n=2)$, duodenum $(n=2)$, breast $(n=2)$, kidney $(n=2)$, and other regions $(n=9$ patients; $12 \%)$. The total radiation dose range was $48-64$ Gy in 3, 4, or 8 equal fractions. Nine lesions $(7.9 \%)$ were treated with 3 fractions, 80 lesions (70.2\%) with 4 fractions, and 25 lesions (21.9\%) with 8 fractions. The median follow-up period was 21 months (range, 7-43) and 16 patients died during follow-up.
Table 1 Patient and lesion characteristics

\begin{tabular}{|c|c|c|}
\hline & $\begin{array}{l}\text { Patient total } \\
(n=76)\end{array}$ & $\begin{array}{l}\text { Lesion total } \\
(n=114)\end{array}$ \\
\hline Age, Median (Range), years & $69(37-91)$ & \\
\hline \multicolumn{3}{|l|}{ Gender, n (\%) } \\
\hline Male & $44(58 \%)$ & \\
\hline Female & $32(42 \%)$ & \\
\hline \multicolumn{3}{|l|}{ ECOG PS, n (\%) } \\
\hline 0 & $42(55 \%)$ & \\
\hline $1-2$ & $34(45 \%)$ & \\
\hline Single lesion, n (\%) & $57(75 \%)$ & \\
\hline \multicolumn{3}{|l|}{ Histology of primary lesion, n (\%) } \\
\hline CRC & $46(61 \%)$ & $73(64 \%)$ \\
\hline Non CRC & $30(39 \%)$ & $41(36 \%)$ \\
\hline Previous local therapy, n (\%) & $8(11 \%)$ & \\
\hline Previous chemotherapy, n (\%) & $52(68 \%)$ & $83(73 \%)$ \\
\hline Posterior chemotherapy, n (\%) & $19(25 \%)$ & $30(26 \%)$ \\
\hline \multicolumn{3}{|l|}{ Metastatic location, n (\%) } \\
\hline Lung & & $70(61 \%)$ \\
\hline Liver & & $44(39 \%)$ \\
\hline MTD, Median (Range), mm & & $19(5-57)$ \\
\hline GTV, Median (Range), ml & & $3.2(0.2-106.8)$ \\
\hline PTV dose BED 10 , Median (Range), Gy & & $150(81.3-180)$ \\
\hline
\end{tabular}

ECOG Eastern Cooperative Oncology Group, PS performance status, CRC colorectal cancer, MTD maximum tumor diameter, GTV gross tumor volume, PTV planning target volume, BED biological effective dose

Death was related to development of cancer in $11 \mathrm{pa}-$ tients and other disease in 1 patient. The cause of death was unknown in the remaining 4 patients. The 1- and 2year OS rates were 96\% (95\% CI: $91-100)$ and 76\% (95\% CI: 64-90), respectively (Fig. 1a).

Seven of the 70 lung lesions and 10 of the 44 liver lesions experienced local failure. The 1- and 2-year LC rates were 89\% (95\% CI: 83-95) and 78\% (95\% CI: 6989), respectively (Fig. 1b). Predictive factors for LC are provided in Table 2. Cut-off value of GTV was defined as $7 \mathrm{ml}$ by ROC analysis. Smaller GTV and addition of post SBRT chemotherapy were significant predictive factors for better local control $(p=0.005, p=0.047)$. New metastatic lesions appeared in 51 patients. The 1- and 2year PFS rates were 38\% (95\% CI: $28-51$ ) and 20\% (95\% CI: 12-34), respectively (Fig. 1c). Predictive factors for PFS and OS are provided in Table 3. The presence of a single metastatic lesion was a significant factor of improved PFS $(p=0.010)$ and addition of post SBRT chemotherapy conferred to better OS $(p=0.078)$. Of the 19 patients received post SBRT chemotherapy, 18 were colorectal cancer patients. So, additional analyses were carried out in 46 colorectal cancer patients. The results of univariate and multivariate analyses shown only post 

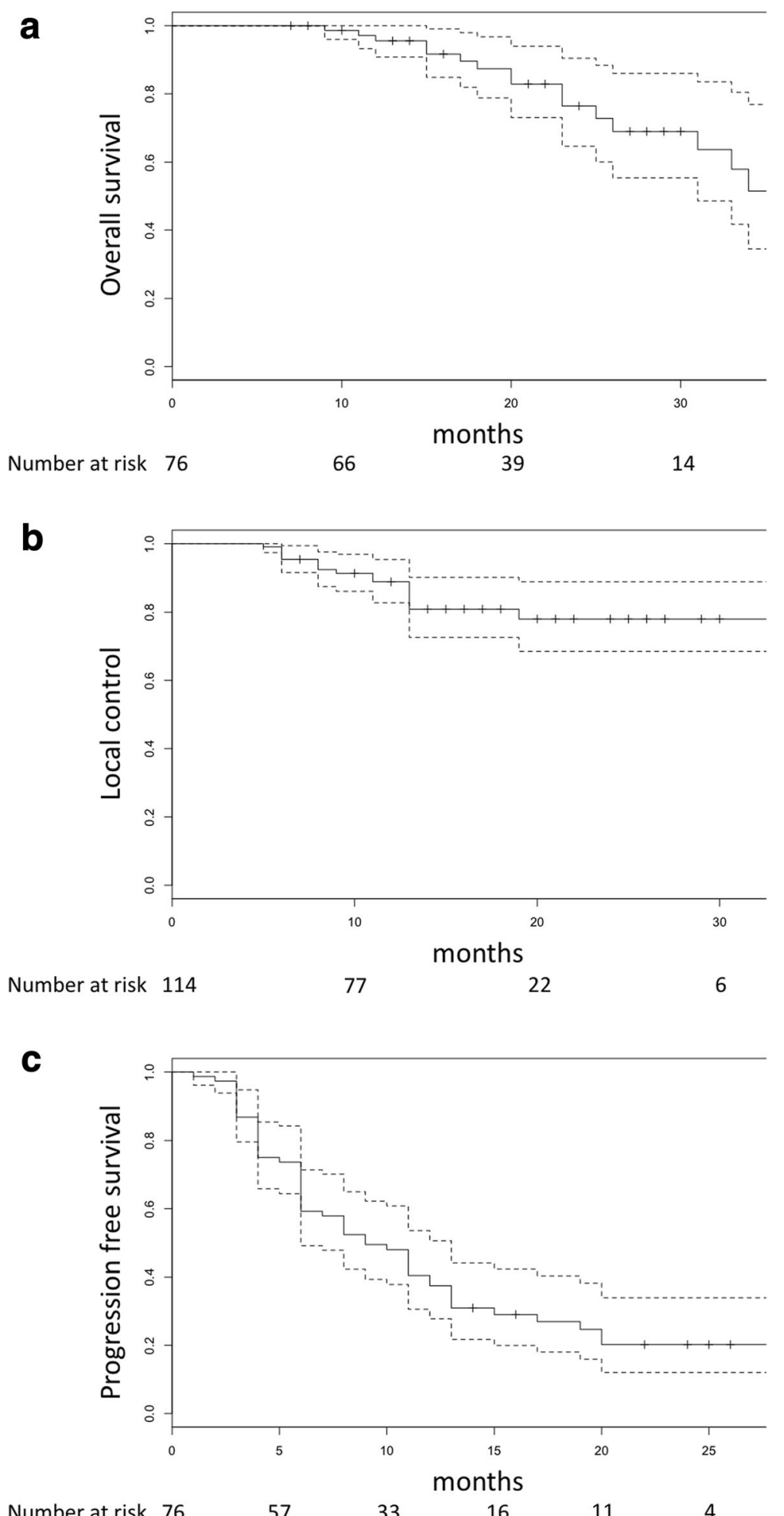

Fig. 1 Kaplan-Meier curves of (a) overall survival, (b) local control, (c) progression free survival

SBRT chemotherapy was significantly associated with better OS among colorectal cancer patients $(p=0.007$ and $p=0.025$, respectively).
Grade 3 radiation dermatitis was observed in 1 patient. This patient underwent two courses of SBRT for liver lesions and dermatitis caused by overlap of two radiation 
Table 2 The univariate and multivariate analysis of factors related to local control

\begin{tabular}{|c|c|c|c|}
\hline & \multicolumn{3}{|l|}{$\underline{\text { Local control }}$} \\
\hline & Univariate ( $p$ value) & Multivariate ( $p$ value) & Hazard ratio $(95 \% \mathrm{Cl})$ \\
\hline Histology: CRC vs others & 0.556 & & \\
\hline Previous chemotherapy: yes vs no & 0.406 & & \\
\hline Posterior chemotherapy: yes vs no & 0.020 & 0.047 & $0.13(0.02-0.97)$ \\
\hline Location: lung vs liver & 0.044 & 0.569 & $0.74(0.25-2.13)$ \\
\hline MTD: $\geq 21$ vs < 21 & 0.010 & & \\
\hline GTV: $\geq 7$ vs $<7$ & 0.002 & 0.005 & $4.62(1.57-13.58)$ \\
\hline PTV BED 10 dose: $95 \geq$ vs $<95$ & 0.582 & & \\
\hline
\end{tabular}

$C R C$ colorectal cancer, MTD maximum tumor diameter, GTV gross tumor volume, PTV planning target volume, BED biological effective dose, $C I$ confidence interval

fields. Overlapped skin dose was total 56 Gy in 4 fractions. Of the 28 patients who underwent more than 2 courses of SBRT, over grade 2 adverse event due to radiation dose overlap was observed only this case. Grade 2 adverse events were observed in 2 patients. One developed chest wall pain. This patient underwent 2 courses of SBRT for lung lesions, but there was no overlap of dose distribution in the chest wall. Another developed radiation pneumonitis. This patient underwent only 1 course of SBRT. Grade 1 adverse events were observed in 53 patients. Regarding toxicities that were related to the method of fiducial marker placement, one grade 1 intra-pelvic hematoma and one stomachache were observed.

\section{Discussion}

We reported a single-institutional retrospective study that investigated and analyzed patients with oligorecurrent lesions in the lungs and liver treated with SBRT using a robotic radiosurgery system. For the 114 lesions of 76 patients treated, the 1- year LC, PFS and OS rates were 89,38 and $96 \%$, respectively. Grade 3 and higher-grade toxicity occurred in only $1 \%(2 / 76)$ of the patients. These results were almost identical to the results reported in other literature (Table 4).

Our results indicated that the LC rate of large GTV $(\geq 7 \mathrm{ml})$ lesions were significantly lower than those of small GTV $(<7 \mathrm{ml})$ lesions $(p=0.005)$. The relationship between tumor volume and LC has been reported previously $[10,11]$. Other reports described primary histology and dosimetric factors as being related to LC. Ahmed et al. described the 1- and 2-year LC rates for colorectal lesions were 79 and $59 \%$, compared with $100 \%$ for noncolorectal lesions [12]. Andratschke et al. described the 2-year LC rates for PTV prescription BED10 > 86.1 Gy was $96.6 \%$ and for $\leq 86.1$ Gy10 $68.1 \%$, the difference being significant [13]. In our study, significant relationship was not observed between histology of CRC and LC. Furthermore, it is difficult to accurately evaluate the exact dosimetric levels because different dose calculation algorithms are used for the lungs and liver treatment.

In this study, PFS was shown to be poor compared to superior LC and OS. This tendency was recognized in other reports as well. The 1- and 2-year PFS rates were $24-51$ and $18-46 \%$ in other reports, respectively [13-20]. Only $12 \%(9 / 76)$ of the patients did not exhibit disease progression or death 20 months after SBRT in this study; these patients all harbored a single metastatic lesion at the time of SBRT consultation. The presence of a single metastatic lesion was a significant predictive factor of improved PFS. However, many of the patients with a single metastatic lesion (48/57) experienced disease progression. This may be due to the fact that patients with the state of systemic disease were included in the treatment group.

Table 3 The univariate and multivariate analysis of factors related to progression free survival and overall survival

\begin{tabular}{|c|c|c|c|c|c|c|}
\hline & \multicolumn{3}{|c|}{ Progression free survival } & \multicolumn{3}{|c|}{ Overall survival } \\
\hline & $\begin{array}{l}\text { Univariate } \\
\text { ( } p \text { value) }\end{array}$ & $\begin{array}{l}\text { Multivariate } \\
\text { ( } p \text { value) }\end{array}$ & $\begin{array}{l}\text { Hazard ratio } \\
(95 \% \mathrm{Cl})\end{array}$ & $\begin{array}{l}\text { Univariate } \\
\text { ( } p \text { value) }\end{array}$ & $\begin{array}{l}\text { Multivariate } \\
\text { ( } p \text { value) }\end{array}$ & $\begin{array}{l}\text { Hazard ratio } \\
(95 \% \mathrm{Cl})\end{array}$ \\
\hline Age: $\geq 70$ vs $<70$ & 0.195 & & & 0.638 & & \\
\hline Gender: male vs female & 0.607 & & & 0.774 & & \\
\hline ECOG PS: 0 vs 1-2 & 0.435 & & & 0.866 & & \\
\hline Lesion number: single vs multiple & 0.008 & 0.010 & $0.46(0.26-0.83)$ & 0.053 & 0.112 & $0.43(0.15-1.22)$ \\
\hline Histology: CRC vs others & 0.496 & & & 0.935 & & \\
\hline Previous chemotherapy: yes vs no & 0.378 & & & 0.927 & & \\
\hline Posterior chemotherapy: yes vs no & 0.088 & 0.126 & $1.60(0.88-2.93)$ & 0.035 & 0.078 & $2.72(0.90-8.27)$ \\
\hline
\end{tabular}

ECOG Eastern Cooperative Oncology Group, PS performance status, CRC colorectal cancer, Cl confidence interval 
Table 4 Recent reports on stereotactic body radiation therapy for lung or liver metastases

\begin{tabular}{|c|c|c|c|c|c|c|c|c|c|c|c|c|}
\hline \multirow[t]{2}{*}{ Aurhor (reference) } & \multirow{2}{*}{$\begin{array}{l}\text { Lesion } \\
\text { /Patients, n }\end{array}$} & \multirow{2}{*}{$\begin{array}{l}\text { CRC } \\
\text { rate, \% }\end{array}$} & \multirow{2}{*}{$\begin{array}{l}\text { Median } \\
\text { f/u period, } m\end{array}$} & \multirow{2}{*}{$\begin{array}{l}\text { Median } \\
\text { MTD, mm }\end{array}$} & \multirow{2}{*}{$\begin{array}{l}\text { Median } \\
\text { GTV, mL }\end{array}$} & \multicolumn{2}{|c|}{ LC, \% } & \multicolumn{2}{|c|}{ PFS, \% } & \multicolumn{2}{|c|}{ OS, $\%$} & \multirow{2}{*}{$\begin{array}{l}A E \geq G 3 \\
\%\end{array}$} \\
\hline & & & & & & $1 \mathrm{yr}$ & $2 \mathrm{yr}$ & $1 \mathrm{yr}$ & $2 \mathrm{yr}$ & $1 \mathrm{yr}$ & $2 \mathrm{yr}$ & \\
\hline Rusthoven (7) & $63 / 38$ & 24 & 15 & NA & 4.2 & 100 & 96 & NA & NA & NA & 39 & 8 \\
\hline Rurthoven (8) & $63 / 47$ & 32 & 16 & 27 & 14.9 & 95 & 92 & NA & NA & NA & 30 & 2 \\
\hline Binkley (10) & $122 / 77$ & 21 & 22 & NA & 3.7 & 91 & 84 & NA & 25 & 94 & 75 & 0 \\
\hline Andratschke (13) & $91 / 52$ & 42 & 17 & 28 & NA & NA & 82 & 35 & 18 & 70 & 45 & $1.9(\geq \mathrm{G} 2)$ \\
\hline Ricard (14) & $77 / 61$ & 21 & 20 & NA & NA & NA & 89 & NA & 32 & NA & 67 & 2 \\
\hline Filippi (15) & $59 / 40$ & 100 & 46 & 15 & NA & 93 & NA & 49 & 27 & 84 & 73 & $7.5(\geq \mathrm{G} 2)$ \\
\hline Agolli (16) & $69 / 44$ & 100 & 36 & 14 & NA & NA & NA & NA & 20 & NA & 68 & 0 \\
\hline Katz (17) & $74 / 69$ & 29 & 15 & 27 & NA & 76 & 57 & 24 & NA & 78 & 37 & 0 \\
\hline Scorsetti (18) & $52 / 42$ & 100 & 24 & 35 & NA & 95 & 91 & NA & 46 & NA & 65 & 0 \\
\hline Joo (19) & $103 / 70$ & 100 & 34 & 29 & NA & 93 & 89 & NA & 35 & NA & 75 & NA \\
\hline Wang (25) & $134 / 95$ & 18 & 17 & NA & 14.6 & 98 & 91 & 51 & 29 & 83 & 61 & 3 \\
\hline Vautrauers-Dewas (26) & $62 / 42$ & 67 & 14 & 25 & 13 & 90 & 86 & NA & NA & 94 & 48 & 6.3 \\
\hline Present study & $114 / 76$ & 61 & 21 & 19 & 3.2 & 89 & 78 & 38 & 20 & 96 & 76 & 1.3 \\
\hline
\end{tabular}

CRC colorectal cancer, MTD maximum tumor diameter, GTV gross tumor volume, LC local control, PFS progression free survival, OS overall survival, AE adverse event, NA:

Furthermore, a Japanese prospective study of resection for oligometastases of non-small cell lung cancer included $18 \%$ (6/34) non-malignant lesions [21]. There may be limits to imaging-guided evaluation for oligometastatic conditions. Recently, studies have indicated that biomarkers are useful for the evaluation of tumor burden [22, 23]. The determination of molecular markers to distinguish oligometastatic from polymetastatic diseases are ongoing [24]. Wong et al. described a candidate classifier using expression levels of three microRNAs (miR-23b, miR-449a, and miR-449b) to predict survival after oligometastasis-directed SBRT [25]. Although still in the research stage, evaluation of oligometastatic conditions using liquid biopsy is expected.

There was a report that patients who received chemotherapy before SBRT had better local control [26]. On the other hand, other reports described contrary results [27, 28]. Klement et al. explained prior chemotherapy affect determination of target volume or reduction of radiation sensitivity [27]. In our study, prior chemotherapy didn't contribute tumor control, but additional chemotherapy after SBRT related to better LC and OS. Particularly, additional chemotherapy after SBRT improved OS among colorectal cancer patients. The strategy of combining surgery with chemotherapy is adopted for liver metastases in colorectal cancer [29]. This strategy may also be applicable to radiotherapy. However, patients who received chemotherapy for metastatic condition had heterogeneity regarding chemotherapy regimen or number of chemotherapy courses. Further analysis is needed to clarify efficacy of combination therapy of chemotherapy and SBRT using a larger number of more homogeneous patients.
Limitations of this study include its retrospective nature and relatively limited follow-up time. Longer follow-up periods are needed for slow-growing tumor subtypes. Additionally, this study was limited by the heterogeneity of tumor histology. The combined analysis of different types of tumor histology with various prior treatments makes thorough evaluation of treatment efficacy difficult.

\section{Conclusions}

SBRT is a safe and effective treatment for patients with lung and liver oligo-recurrence. Additional chemotherapy after SBRT improved local control, and single metastatic lesion was a significant predictive factor of better PFS in this study. Among colorectal cancer patients, additional chemotherapy after SBRT significantly associated better OS.

\section{Abbreviations}

Cls: Confidence intervals; CT: Computed tomography; FDG-PET: ${ }^{18} \mathrm{~F}-$ fluorodeoxyglucose positron emission tomography scans; GTV: Gross tumor volume; LC: Local control; MR: Magnetic resonance; OS: Overall survival; PFS: Progression free survival; PTV: Planning target volume; SBRT: Stereotactic body radiation therapy

\section{Acknowledgments}

We wish to thank Editage (www.editage.jp) for English language editing.

\section{Availability of data and materials}

The datasets used and/or analyzed during the current study are available from the corresponding author on reasonable request.

\section{Authors' contributions}

$\mathrm{MN}$ : made substantial contributions to the conception and design of the study. MN: aided in the collection of data, statistical analysis, and drafting of the manuscript. $\mathrm{NH}, \mathrm{HM}, \mathrm{HU}, \mathrm{AH}, \mathrm{RN}, \mathrm{YM}$, and HK: assisted the data acquisition. $\mathrm{HN}$ : helped to draft the manuscript. All authors confirm that they have read and approved the final version. 


\section{Ethics approval and consent to participate}

All study participants provided informed written consent. The study protocol was approved by the Research Ethics Committee of our institution (reference number: 2018-[kenkyu01]-09). The research was conducted in accordance with the 1964 Declaration of Helsinki and its later amendments.

\section{Consent for publication}

All study participants provided informed written consent for publication.

\section{Competing interests}

The authors declare that they have no competing interests.

\section{Publisher's Note}

Springer Nature remains neutral with regard to jurisdictional claims in published maps and institutional affiliations.

\section{Author details}

'Department of Radiation Oncology, Kobe Minimally invasive Cancer Center, 8-5-1, Minatojima Nakamachi, Chuo-ku, Kobe, Hyogo 650-0046, Japan. ${ }^{2}$ Division of Radiation Oncology and Particle Therapy, National Cancer Center Hospital East, 6-5-1, Kashiwanoha, Kashiwa, Chiba 277-8577, Japan. ${ }^{3}$ Department of Radiation Oncology, University of Florida Proton Therapy Institute, 2015 N Jefferson St, Jacksonville, FL 32206, USA. ${ }^{4}$ Division of Radiation Oncology, Kobe University Graduate School of Medicine, 7-5-2 Kusunoki-Cho, Chuo-ku, Kobe, Hyogo 650-0017, Japan. ${ }^{5}$ Department of Radiology, Hyogo Ion Beam Medical Center, 1-2-1, Koto, Shingu-cho, Tatsuno, Hyogo 679-5165, Japan

Received: 1 March 2018 Accepted: 16 April 2018

Published online: 23 April 2018

\section{References}

1. Redig AJ, McAllister SS. Breast cancer as asystemic disease: a view of metastasis. J Intern Med. 2013;274:113-26.

2. Hellman S, Weichselvaum RR. Oligometastases. J Clin Oncol. 1995:13:8-10.

3. Tree AC, Khoo VS, Eeles PA, et al. Stereotactic body radiotherapy for oligometastases. Lancet Oncol. 2013;14:28-37.

4. Yamashita $H$, Niibe $Y$, Yamamoto $T$, et al. Lung stereotactic radiotherapy for oligometastases: comparison of oligo-recurrence and sync-oligometastases. Jpn J Clin Oncol. 2016;46:687-91.

5. Nordlinger B, Guiguet M, Vaillant JG, et al. Surgical resection of colorectal carcinoma metastases to the liver. Cancer. 1996:77:1254-62.

6. Pfannschmidt J, Dienemann $\mathrm{H}, \mathrm{Hoffmann} \mathrm{H}$. Surgical resection of pulmonary metastases from colorectal cancer: a systematic review of published series. Ann Thorac Surg. 2007;84:324-38.

7. Rusthoven KE, Kavanagh BD, Burri SH, et al. Multi-institutional phase I/II trial of stereotactic body radiation therapy for lung metastases. J Clin Oncol. 2009:27:1579-84.

8. Rusthoven KE, Kavanagh BD, Cardenes $\mathrm{H}$, et al. Multi-institutional phase I/II trial of stereotactic body radiation therapy for liver metastases. J Clin Oncol. 2009;27:1572-7.

9. Nakamura M, Nishimura H, Nakayama M, et al. Dosimetric factors predicting radiation pneumonitis after CyberKnife stereotactic body radiotherapy for peripheral lung cancer. Br J Radiol. 2016;89:1068

10. Binkley MS, Trakul N, Jacobs LR, et al. Colorectal histology is associated with an increased risk of local failure in lung metastases treated with stereotactic ablative radiation therapy. Int J Radiat Oncol Biol Phys. 2015;92:1044-52.

11. Bae SH, Kim MS, Cho CK, et al. High dose stereotactic body radiotherapy using three fractions for colorectal oligometastases. J Surg Oncol. 2012;106: 138-43.

12. Ahmed KA, Caudell JJ, El-Haddad G, et al. Radiosensitivity differences between liver metastases based on primary histology suggest implications for clinical outcomes after stereotactic body radiation therapy. Int J Radiat Oncol Biol Phys. 2016;95:1399-404

13. Andratschke N, Prays A, Stadtfeld S, et al. Clinical results of mean GTV dose optimized robotic guided SBRT for liver metastases. Radiat Oncol. 2016;11:74.

14. Ricardi U, Filippi AR, Guarneri A, et al. Stereotactic body radiation therapy for lung metastases. Lung Cancer. 2012;75:77-81.

15. Filippi AR, Badellino S, Ceccarelli $M$, et al. Stereotactic ablative radiation therapy as first local therapy for lung oligometastases from colorectal cancer: a single-institution cohort study. Int J Radiat Oncol Biol Phys. 2015 91:524-9.

16. Agolli L, Bracci S, Nicosia L, et al. Lung metastases treated with stereotactic ablative radiation therapy in oligometastatic colorectal cancer patients: outcomes and prognostic factors after long-term follow-up. Clin Colorectal Cancer. 2017;16:58-64.

17. Katz AW, Carey-Sampson M, Muhs AG, et al. Hypofractionated stereotactic body radiation therapy (SBRT) for limited hepatic metastases. Int J Radiat Oncol Biol Phys. 2007:67:793-8.

18. Scorsetti M, Comito T, Tozzi A, et al. Final results of a phase II trial for stereotactic body radiation therapy for patients with inoperable liver metastases from colorectal cancer. J Cancer Res Clin Oncol. 2015;141:54353.

19. Joo JH, Park JH, Kim JC, et al. Local control outcomes using stereotactic body radiation therapy for liver metastases from colorectal cancer. Int J Radiat Oncol Biol Phys. 2017;99:876-83.

20. Ahmed KA, Fulp WJ, Berglund AE, et al. Differences between colon cancer primaries and metastases using a molecular assay for tumor radiation sensitivity suggest implications for potential oligometastatic SBRT patient selection. Int J Radiat Oncol Biol Phys. 2015:92:837-42.

21. Endo $C$, Hasumi T, Matsumura $Y$, et al. A prospective study of surgical procedures for patients with oligometastatic non-small cell lung cancer. Ann Thorac Surg. 2014;98:258-64

22. Spindler KL, Pallisgaard N, Vogelius I, Jakobsen A. Quantitative cell-free DNA KRAS, and BRAF mutations in plasma from patients with metastatic colorectal cancer during treatment with cetuximab and irinotecan. Clin Cancer Res. 2012;18:1177-85.

23. Lipson EJ, Velculescu VE, Pritchard TS, et al. Circulating tumor DNA analysis as a real-time method for monitoring tumor burden in melanoma patients undergoing treatment with immune checkpoint blockade. J ImmunoTherapy Cancer. 2014;2:42.

24. Weichselbaum RR, Hellman S. Oligometastases revisited. Nat Rev Clin Oncol. 2011:8:378-82.

25. Wong AC, Watson SP, Pitroda SP, et al. Clinical and molecular markers of long-term survival after oligometastasis-directed stereotactic body radiotherapy (SBRT). Cancer. 2016;122:2242-50.

26. Vautravers-Dewas $C$, Dewas $S$, Bonodeau F, et al. Image-guided robotic stereotactic body radiation therapy for liver metastases: is there a dose response relationship? Int J Radiat Oncol Biol Phys. 2011;81:39-47.

27. Klement RJ, Guckenberger M, Alheid H, et al. Stereotactic body radiotherapy for oligo-metastatic liver disease-Infuence of pre-treatment chemotherapy and histology on local tumor control. Radiother Oncol. 2017;123:227-33.

28. Stera S, Balermpas P, Chan MKH, et al. Breathing-motion-compensated robotic guided stereotactic body radiation therapy. Strahlenther Onkol. 2018;194:143-55.

29. Mitry $E$, Fields LA, Bleiberg $H$, et al. Adjuvant chemotherapy after potentially curative resection of metastases from colorectal cancer: a pooled analysis of two randomized trials. J Clin Oncol. 2008;26:4906-11.

Ready to submit your research? Choose BMC and benefit from

- fast, convenient online submission

- thorough peer review by experienced researchers in your field

- rapid publication on acceptance

- support for research data, including large and complex data types

- gold Open Access which fosters wider collaboration and increased citations

- maximum visibility for your research: over $100 \mathrm{M}$ website views per year

At BMC, research is always in progress.

Learn more biomedcentral.com/submissions 